\title{
Transition Process of Frequencies of Pure Tone Caused by Vortex Shedding in a Pipeline Containing a Double Orifice*
}

\author{
Masashi SANO** and Takeshi OYAIZU*** \\ ** Department of Mechanical Engineering, Shizuoka Institute of Science and Technology \\ 2200-2 Toyosawa, Fukuroi, Shizuoka, 437-8555, Japan \\ E-mail: sano@me.sist.ac.jp \\ ***Kohritsu Co., Ltd.
}

1588 Shimoishida-cho, Hamamatsu, Shizuoka, 453-0006, Japan

\begin{abstract}
Experimental investigations were performed on the generation of resonant sound by flow in a pipeline containing two closely spaced orifice plates. The frequencies of the generated tone were scrutinized as the velocity was increased from 3 to $25 \mathrm{~m} / \mathrm{s}$. To determine the convection velocity of the vortices formed between two plates, measurements were made of the phase angle of the fluctuating velocity using a hot-wire anemometer. The vortex shedding frequency is locked into the pipe modes, but indicates a slight increase with an increase in the flow velocity. The ratio of the distance between the two orifice plates to the spacing of vortices is expressed by $m+\varepsilon$, where $m$ is an integer and $\varepsilon$ is a decimal part. It was found that the value of $\varepsilon$ decreases with increasing flow velocity within a range from 0 to 0.5 , and a change of the number of vortices or a frequency jump occurs when $\varepsilon$ reaches a critical value.
\end{abstract}

Key words: Flow Induced Vibration, Vortex Shedding, Hole Tone, Pure Tone, Double Orifice, Convection Velocity, Impinging Shear Layer, Acoustic Resonance, Pipe Flow

\section{Introduction}

Orifice plates are widely used as convenient pressure-reduction and flow-regulation devices in a pipe flow. For large pressure-reduction a double orifice is applied to avoid excessive turbulent noise and cavitation in a liquid flow that are caused by increasing local velocity due to a decrease in the orifice plate hole diameter. In this case, however, severe pure tone, whose frequency changes stepwise with an increase in velocity, is frequently generated $^{(1)}$.

Chanaud et al. ${ }^{(2)}$ investigated the hole tone produced when an axisymmetric jet issued from a converging nozzle impinges on a flat plate with a hole placed down stream. They suggested that the mechanism of tone generation closely resembles that of the edge tone and showed that the dimensionless frequency (Strouhal number) depends on the distance between the nozzle exit and the downstream plate and changes stepwise with the dimensionless velocity (Reynolds number).

Studies have also been performed on the pure acoustic tone produced in a rectangular duct with two closely spaced baffles. Nomoto et al. ${ }^{(3)}$ conducted experiments on vortices generated between two baffles installed in a duct. They found that stable vortices are formed and pure tones are produced when the frequency of the vortex shedding from the baffle 
coincides with the longitudinal natural frequency of the duct. Hourigan et al. ${ }^{(4)}$ reported experimental and numerical investigations of the generation of resonant sound by flow, and sound field feedback was calculated by the finite element method, and a discrete-vortex model was used to predict the observed separated flow. For a system where two orifice plates were installed in a circular pipe, Harris et al. ${ }^{(5)}$ examined the effects of the orifice hole diameter and orifice plate spacing on a frequency of sound generated over a wide velocity range. Lamoureux et al. ${ }^{(6)}$ experimented on the effects of with/without converging the section of the first orifice inlet or glass wool attached inside the pipe on the frequency and the acoustic pressure of the generated sound. Huang et al. ${ }^{(7)}$ successfully achieved active control of produced sound by passing signals of the acoustic pressure and velocity fluctuations in the pipe through a phase shifter and an amplifier to a loudspeaker at the pipeline entrance. Despite these investigations the convection velocity of vortices and vortex spacing, which dominate the frequency of generated sound, namely, the Strouhal number under an acoustic resonance condition, remain unclarified.

In this paper experiments are carried out on the effects of velocity and orifice spacing on the frequency of sound generated by flow in a pipeline containing two closely spaced sharp-edged orifice plates. Additionally the convection velocity of vortices is examined at typical flow velocity by measuring a phase angle of the fundamental fluctuating velocity component. Based on these results, the frequency transition process is elucidated when the frequency of sound due to vortex shedding is locked into an acoustic resonance mode.

\section{Nomenclature}

$a \quad$ : speed of sound

$D$ : internal diameter of pipe $(=50 \mathrm{~mm})$

$d \quad$ : hole diameter of orifice plate $(=25 \mathrm{~mm})$

$f \quad$ : frequency

$f_{n} \quad$ : n-th order acoustic natural frequency of pipe mode

$h \quad:$ thickness of orifice plate $(=3 \mathrm{~mm})$

$j \quad$ : imaginary unit

$L \quad$ : pipe length

$l \quad:$ distance between orifice plates

$m$ : stage number $(=1,2, \cdots)$

Re : Reynolds number $=U_{m} d / \mathrm{v}$

$r$ : radial coordinate

$S P L:$ sound pressure level

St $:$ Strouhal number $=f \cdot(l+h) / U_{m}$

$U$ : time-mean velocity

$U_{c}$ : convection velocity of vortices

$U_{m}$ : cross-sectional averaged velocity

$U_{\text {max }}$ : maximum value of $U$

$u^{\prime}:$ rms value of fluctuating velocity

$u_{1}^{\prime}:$ rms value of fundamental component of fluctuating velocity

$x \quad$ : longitudinal coordinate

$\lambda$ : spacing of vortices

$v$ : kinematic viscosity of fluid

$\rho \quad:$ density of fluid

$\psi$ : phase lag of fundamental component of fluctuating velocity 


\section{Apparatus and method of experiments}

Figure 1 shows the schematic outline of the experimental apparatus. Air was introduced to the test section made of a brass pipe with an internal diameter of $50 \mathrm{~mm}$ and a total length of $635 \mathrm{~mm}$ and exhausted by a centrifugal blower after passing through a settling chamber of $0.34 \mathrm{~m}^{3}$ and an orifice flow meter. As shown in Fig. 2, a pair of single-hole orifice plates was installed in the tested pipe. The distance between the first plate and the pipe inlet was kept constant at $132 \mathrm{~mm}$, and three different plate spacings $l$ of 17,32 , and $47.5 \mathrm{~mm}$ were employed. The orifice plates were made of brass with squared edges, and hole diameter $d$ and thickness $h$ were 25 and $3 \mathrm{~mm}$, respectively.

The velocity was controlled carefully by changing the rotation speed of the blower. Generated noise was measured using a condenser microphone set $150 \mathrm{~mm}$ horizontally from the pipe center. An I-type hot wire probe was used for velocity measurement downstream of the first plate. An FFT analyzer was used to obtain the peak frequency, its amplitude and phase angle of the noise generated, and fluctuating velocity. To obtain the acoustic natural frequencies of the pipe mode, the sound pressure inside the pipe produced by a loud speaker located $300 \mathrm{~mm}$ upstream of the pipe inlet was measured using a condenser microphone. The frequency resolution in the fast Fourier transform was $1.25 \mathrm{~Hz}$ at $f \leq 500 \mathrm{~Hz}$ and 2.5 $\mathrm{Hz}$ at $500 \mathrm{~Hz}<f \leq 1000 \mathrm{~Hz}$.

Flow visualization was performed by mixing white smoke in the flow between the two orifice plates and illuminating by a sheet of light from a stroboscope. For this purpose the test section was made of acrylic resin.

\section{Theory of acoustic natural frequency}

\subsection{Transfer matrix of piping system}

In the pipeline containing a double orifice, five straight pipe elements, from (1) to (5) shown in Fig. 3, can be regarded as connected in a series. Neglecting the fluid viscosity, the relationship between pressure fluctuation $p$ and flow fluctuation $Q$ at the pipe inlet (subscript in) and the pipe outlet (subscript out) can be expressed as

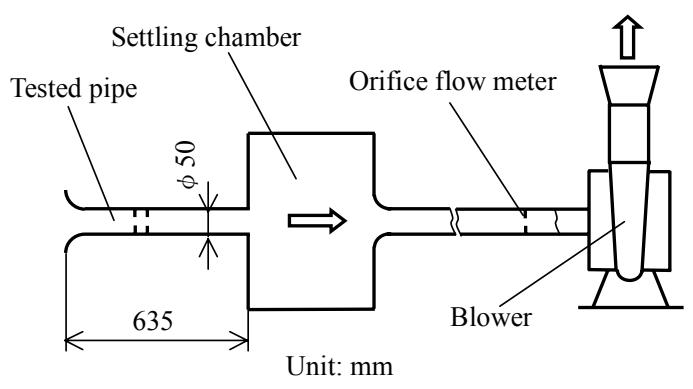

Fig. 1 Arrangement of experimental apparatus

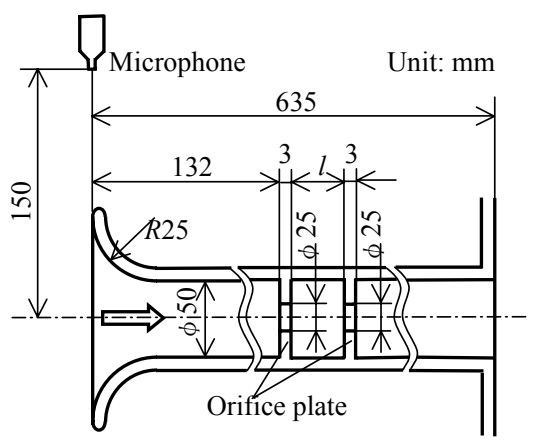

Fig. 2 Details of test section 


$$
\left[\begin{array}{l}
p_{\text {in }} \\
Q_{\text {in }}
\end{array}\right]=M_{1} \cdot M_{2} \cdots M_{5}\left[\begin{array}{l}
p_{\text {out }} \\
Q_{\text {out }}
\end{array}\right]=\left[\begin{array}{ll}
A & B \\
C & D
\end{array}\right]\left[\begin{array}{l}
p_{\text {out }} \\
Q_{\text {out }}
\end{array}\right],
$$

where $M_{i}(i=1,2, \cdots, 5)$ is a transfer matrix of each pipe element given by the following equation and $A, B, \mathrm{C}$, and $D$ are the elements of the $2 \times 2$ matrix derived from the product of each transfer matrix:

$$
M_{i}=\left[\begin{array}{ll}
A_{i} & B_{i} \\
C_{i} & D_{i}
\end{array}\right]=\left[\begin{array}{lr}
\cos (2 \pi f / a) L_{i} & j Z c_{i} \sin (2 \pi f / a) L_{i} \\
j\left(1 / Z c_{i}\right) \sin (2 \pi f / a) L_{i} & \cos (2 \pi f / a) L_{i}
\end{array}\right],
$$

where $L_{i}$ is the pipe length including the open-end correction and $Z c_{i}$ is expressed by

$$
Z c_{i}= \begin{cases}\rho a /(\pi / 4) D^{2} & (i=1,3,5) \\ \rho a /(\pi / 4) d^{2} & (i=2,4) .\end{cases}
$$

The boundary conditions are

$$
p_{\text {in }}=p_{\text {out }}=0 .
$$

Thus natural frequencies $f_{n}$ are given by $f$, which satisfies the following equation:

$$
\begin{aligned}
B=\{ & {\left[\left(A_{1} A_{2}+B_{1} C_{2}\right) A_{3}+\left(A_{1} B_{2}+B_{1} D_{2}\right) C_{3}\right] A_{4}+\left[\left(A_{1} A_{2}+B_{1} C_{2}\right) B_{3}\right.} \\
& \left.\left.+\left(A_{1} B_{2}+B_{1} D_{2}\right) D_{3}\right] C_{4}\right\} B_{5}+\left\{\left[\left(A_{1} A_{2}+B_{1} C_{2}\right) A_{3}+\left(A_{1} B_{2}+B_{1} D_{2}\right) C_{3}\right] B_{4}\right. \\
& \left.+\left[\left(A_{1} A_{2}+B_{1} C_{2}\right) B_{3}+\left(A_{1} B_{2}+B_{1} D_{2}\right) D_{3}\right] D_{4}\right\} D_{5}=0 .
\end{aligned}
$$

\subsection{Calculation results}

Table 1 shows the acoustic natural frequencies (for order $n$ of 1 to 4 ) of the pipe mode obtained from Eq. (5) for orifice spacing $l$ of $47.5 \mathrm{~mm}$. Since a preliminary experiment showed that the open-end correction is $0.2 D, 0.4 D$, and $0.2 d$ for the pipe inlet, the pipe outlet, and the orifice plate hole, respectively, these values were added to the actual pipe length in the calculation. As seen from Table 1, the difference between the above theory and the experiment is less than $1.0 \%$.

The acoustic natural modes of tested pipe, which correspond to Table 1 given by transfer matrix method ${ }^{(8)}$, are illustrated in Fig. 4. In this figure, abscissa $x$, which indicates a distance along the pipe from the upstream surface of the first orifice plate, is positive with the flow direction. The vertical axis, which denotes pressure fluctuation amplitudes $|p|$, is normalized using its maximum value.

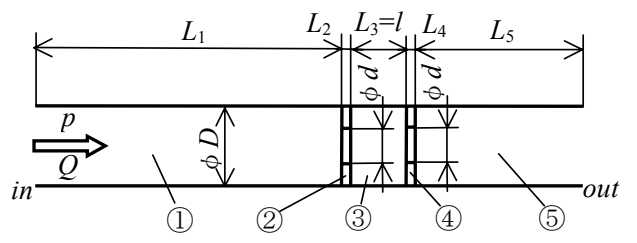

Fig. 3 Piping system containing orifice plates

Table 1 Acoustic natural frequencies of pipeline at $l=47.5 \mathrm{~mm}$

\begin{tabular}{c|c|c}
\hline \hline Order $n$ & Theory $(\mathrm{Hz})$ & Experiment $(\mathrm{Hz})$ \\
\hline 1 & 251.1 & 248.75 \\
2 & 520.1 & 515.0 \\
3 & 748.4 & 750.0 \\
4 & 989.4 & 980.0 \\
\hline
\end{tabular}




\section{Experimental results and discussions}

\subsection{Sound pressure and fluctuating velocity accompanying vortex shedding}

Figures 5(a) and (b) exhibit the frequency spectra of the sound pressure and the fluctuating velocity measured just downstream from the first plate, respectively, when the velocity averaged over cross section $U_{m}$ at the plate hole is $5.84 \mathrm{~m} / \mathrm{s}\left(R e=9.83 \times 10^{3}\right)$ and distance $l$ between the two orifice plates is $47.5 \mathrm{~mm}$. In these figures the sound pressure component of $250 \mathrm{~Hz}$ is predominant, and a distinct peak of the fluctuating velocity is produced at the same frequency as the sound pressure. Removing the second orifice plate, as plotted by broken lines in Fig. 5, the peaks of both sound pressure and fluctuating velocity at $250 \mathrm{~Hz}$ disappear. Therefore it is found that a component of $250 \mathrm{~Hz}$ is generated by two closely spaced orifice plates. The following discussions concentrate on the maximum components of sound pressure.

\subsection{Jet flow velocity and sound pressure}

Frequency $f$ as well as sound pressure level SPL of the generated noise are shown in Fig. 6 as a function of the mean velocity at the hole for plate spacing of $l=47.5 \mathrm{~mm}$. As the velocity gradually increases beyond $3.0 \mathrm{~m} / \mathrm{s}$, the frequency indicates a slight increase along the first acoustic natural frequency $f_{1}$, as shown by symbol $\bigcirc$, except to decrease
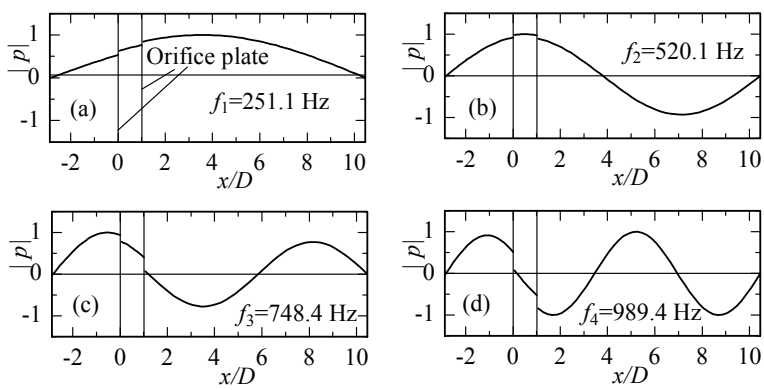

Fig. 4 Acoustic natural modes of pipeline at $l=47.5 \mathrm{~mm}$
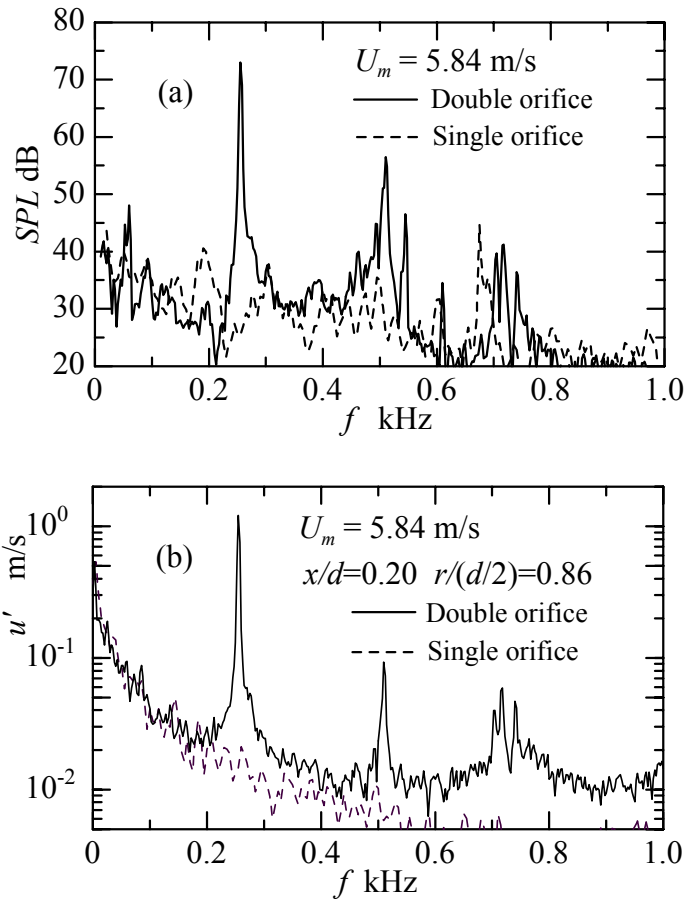

Fig. 5 Spectra: (a) acoustic pressure and (b) fluctuating velocity 
once at $5.1 \mathrm{~m} / \mathrm{s}$ and $8.3 \mathrm{~m} / \mathrm{s}$; afterward at $11.3 \mathrm{~m} / \mathrm{s}$ it jumps to $740 \mathrm{~Hz}$ which almost equals third natural frequency $f_{3}$. Thus the velocity region of less than $11.3 \mathrm{~m} / \mathrm{s}$ is found to be divided into three regions: $3.0 \mathrm{~m} / \mathrm{s} \leq U_{m}<5.1 \mathrm{~m} / \mathrm{s}, \quad 5.1 \mathrm{~m} / \mathrm{s} \leq U_{m}<8.3 \mathrm{~m} / \mathrm{s}$, and $8.3 \mathrm{~m} / \mathrm{s} \leq U_{m}<11.3 \mathrm{~m} / \mathrm{s}$. As explained later in Section 5.4, at each region that corresponds to stage number $m$ of 3,2, and 1 in the order of the velocity, there are different numbers of vortices between the two orifice plates. With further increasing velocity, frequency jumps are repeated from one natural frequency to another. Reviewing Fig. 6 in detail, the frequency increases slightly with an increase in velocity and shows a rapid change along a solid line emanating from the origin just before the transition of frequency. As discussed later in Section 5.4, these solid lines correspond to constant Strouhal numbers of 1.04, 1.94, and 2.84 in the order of inclination.

The sound pressure level repeats its increase and decrease, taking a peak value at each stage. For the decreasing velocity, frequency transition occurred at smaller velocity than the increasing velocity case: a weak hysteresis was observed. In this paper increasing velocity results are presented in the following discussions.

Figures 7 and 8 show the results for $l=32$ and $17 \mathrm{~mm}$, respectively. The features of the changes in frequency and sound pressure level with the velocity resemble those in Fig. 6, but exhibit a trend in which the smaller the spacing between two orifice plates becomes, the higher the frequency becomes and the more the sound pressure increases.

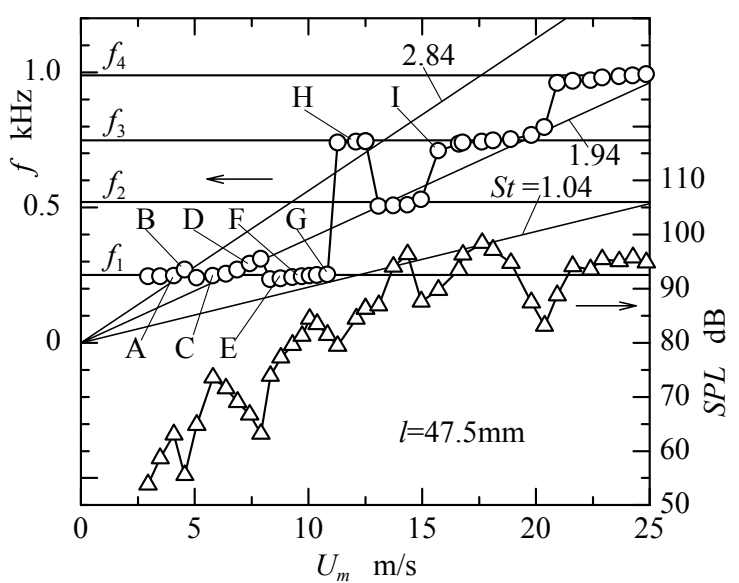

Fig. 6 Dominant frequency and acoustic pressure vs. mean velocity at orifice plate hole at $l=47.5 \mathrm{~mm}$

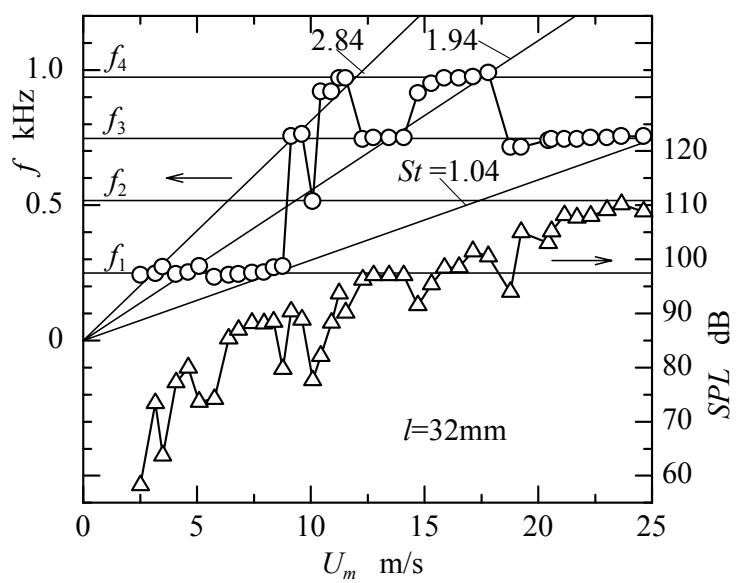

Fig. 7 Dominant frequency and acoustic pressure vs. mean velocity at orifice plate hole at $l=32 \mathrm{~mm}$ 


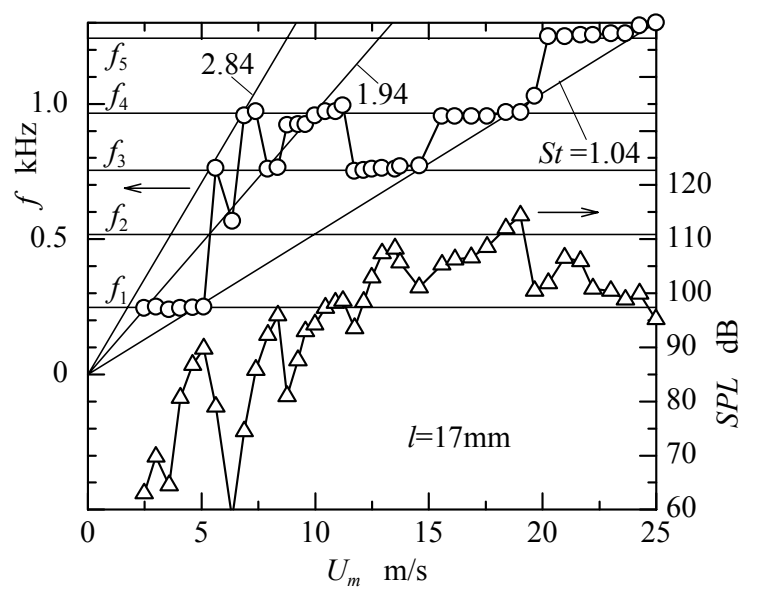

Fig. 8 Dominant frequency and acoustic pressure vs. mean velocity at orifice plate hole at $l=17 \mathrm{~mm}$

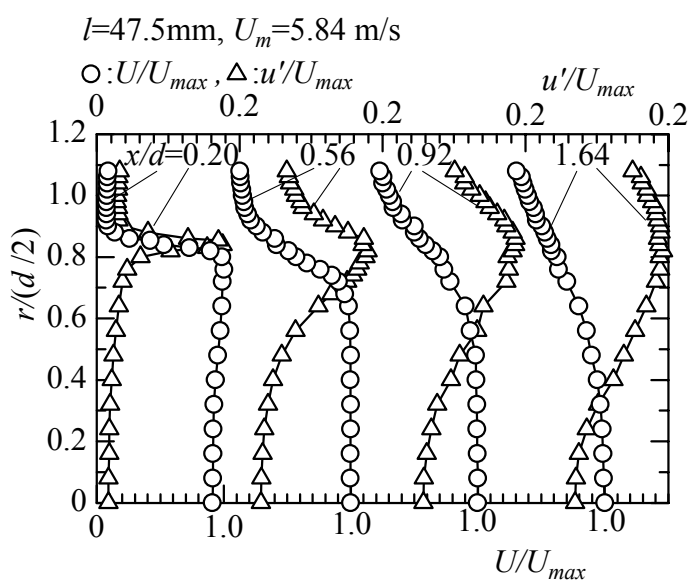

Fig. 9 Distributions of axial velocity and turbulence intensity

\subsection{Time-mean and fluctuating velocity}

The profiles of the time-mean velocity and the turbulence intensity downstream of the first orifice plate measured at $l=47.5 \mathrm{~mm}$ and $U_{m}=5.84 \mathrm{~m} / \mathrm{s}$ are shown in Fig. 9. Just downstream of the first plate vena contracta is generated; at the section of $x / d=0.20$ the velocity shows a concave profile since the pressure at the jet center is higher than at the circumference due to the curved streamlines and as a result the velocity is decreased. However at the downstream section from $x / d=0.56$, the profile near the jet center becomes uniform due to the parallel streamlines. At all sections the turbulence intensity takes maximum at the radial position of $r /(d / 2) \approx 0.86$, where the velocity gradient reaches maximum. In Fig. $9 U_{\max }$ indicates maximum velocity at each section and equals the uniform velocity.

The distributions of the amplitude and the phase lag of the fundamental fluctuating-velocity component $(=250 \mathrm{~Hz})$ are shown in Fig. 10 , where $u_{1}^{\prime}$ is the rms value of the fundamental fluctuating-velocity component and $\psi$ is the phase lag with respect to the sound pressure measured simultaneously as the fluctuating velocity. The phase angle at just the downstream section of the first orifice plate most greatly lags at $r /(d / 2) \approx 0.86$, where the turbulence intensity takes maximum value, and as the section goes downstream it increases gradually while its radial distribution approaches a flat shape.

\subsection{Convection velocity of vortices}

Based on the results shown in Section 5.3, the radial position of the hot-wire probe was fixed at $r /(d / 2)=0.86$ and the axial changes in the phase lag of the fundamental 
fluctuating-velocity component were examined at each velocity corresponding to A to I in Fig. 6. By way of illustration the results for A-G are shown in Fig. 11, where $\psi$ represents the phase lag referenced with respect to $x=0$ and the value of $\psi / 2 \pi$ at $x /(l+h)=1$ expresses the ratio of distance $l+h$ to spacing $\lambda$ of vortices produced between two orifice plates. The values of $(l+h) / \lambda$ obtained from $(\psi / 2 \pi)_{x /(l+h)=1}$ in Fig. 11 are plotted by symbol $\square$ in Fig. 12 as a function of velocity $U_{m}$. This figure also shows the results for orifice spacing $l=32 \mathrm{~mm}$ (symbol $\triangle$ ) and $l=17 \mathrm{~mm}$ (symbol $\bigcirc$ ). As seen from this figure, $(l+h) / \lambda$ can be written as

$$
(l+h) / \lambda=m+\varepsilon,
$$

where $m$ is an integer (stage number) and $\varepsilon$ is a decimal part. In Fig. 12 the experimental data, whose frequency are almost constant and which belong to the same stage as each other, are connected by solid lines. Focusing on these data, $\varepsilon$ decreased as velocity increased. The value of $\varepsilon$ is associated with the summation of the time lag between the impingement of the vortex on the second orifice plate and the occurrence of the sound pulse and the time required for the generation of a vortex seed after the arrival of the sound pulse at the first orifice plate. In addition the decrease in $\varepsilon$ means an increase in the above time $\operatorname{lag}^{(9)}$.

Now the average convection velocity of the vortices is given by the following equation $^{(10)}$ :

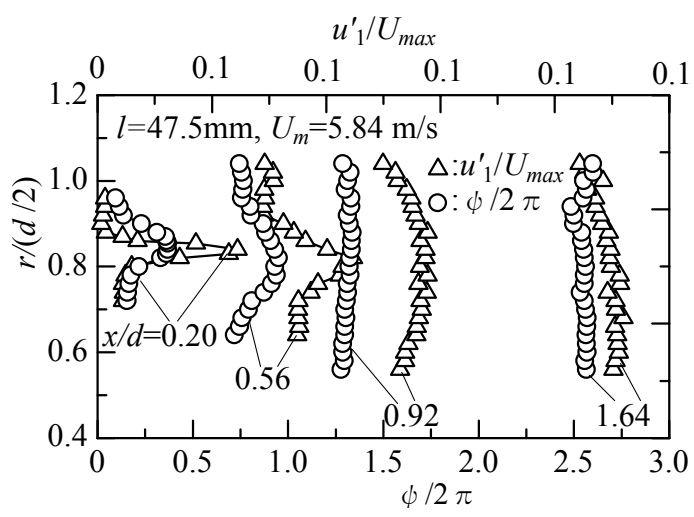

Fig. 10 Distributions of phase-lag and amplitude of fundamental fluctuating-velocity component

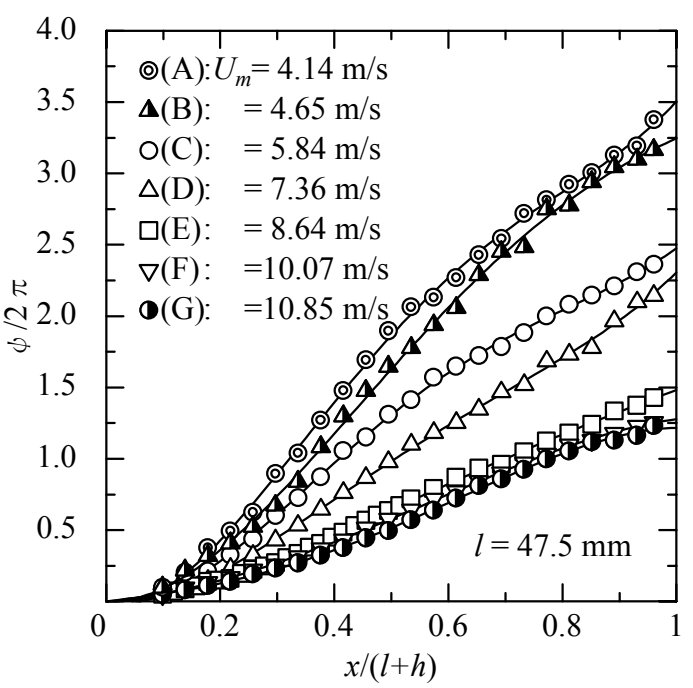

Fig. 11 Phase-lag changes of fundamental fluctuating-velocity component along pipe 


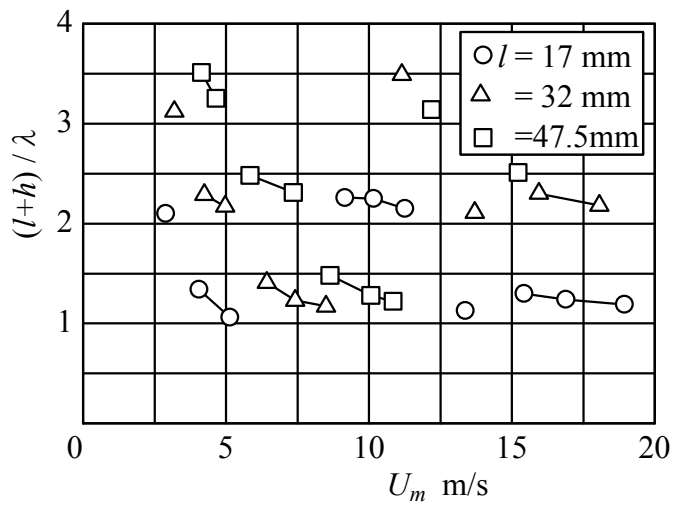

Fig. 12 Ratio of distance between two orifice plates to spacing of vortices vs. mean velocity at orifice plate hole

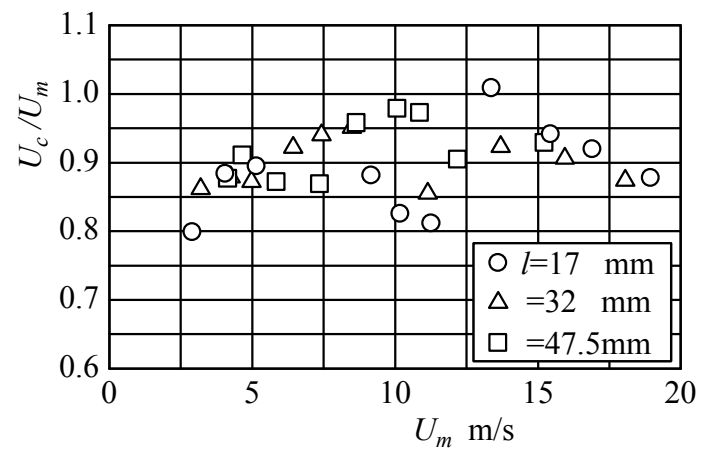

Fig. 13 Convection velocity $U_{c} / U_{m}$ vs. mean velocity at orifice plate hole for different distances between two orifice plates

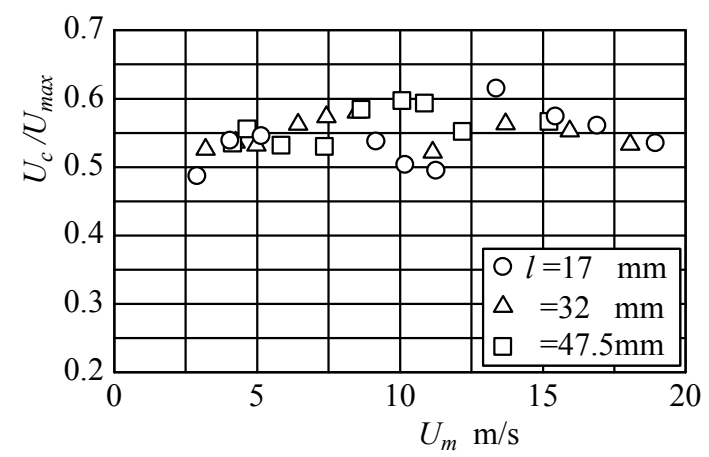

Fig. 14 Convection velocity $U_{c} / U_{\max }$ vs. mean velocity at orifice plate hole for different distances between two orifice plates

$$
U_{c}=\lambda f=\frac{f \cdot(l+h)}{(l+h) / \lambda} .
$$

Figure 13 shows convection velocity $U_{c}$ calculated by using Eq. (7) for the experimental data in Fig. 12. The ratio of vortex convection velocity $U_{c}$ to mean velocity $U_{m}$ is 0.81 to 1.01. In the experiment shown in Fig. 9, $U_{m} / U_{\max }$ was 0.61 . Consequently using this relationship, Fig. 14 is obtained from Fig. 13. Convection velocity $U_{c} / U_{\max }$ takes values between 0.49 and 0.62 , which hardly depend on either orifice spacing $l$ or stage number $m$.

In the past many measurements of vortex convection velocity have been made on the edge tone ${ }^{(11)}$, cavity tone ${ }^{(12)}$, free jet issued from a convergent nozzle ${ }^{(13)-(15)}$, impinging jet on a circular pipe ${ }^{(10)}$, and so on. On the other hand in this experiment the vena contracta is produced and the jet is under the strong influence of the acoustic resonance; nevertheless 
comparison between the results shown in Fig. 14 and the data reported by the above previous studies gives general agreement.

The Strouhal numbers are discussed below. In the present system the sound pulse, which is caused by the impingement of the shear layer separated from the upstream edge of the first orifice on the second orifice, is fed back upstream. Disturbances in the shear layer with discrete frequency develop toward downstream and roll into vortices, and as a result large discrete noise is generated. Therefore the Strouhal number is defined as follows:

$$
S t=\frac{f \cdot(l+h)}{U_{m}} \text {. }
$$

Substituting Eqs. (6) and (7) into Eq. (8) gives

$$
S t=\frac{U_{c}}{U_{m}} \frac{l+h}{\lambda}=\frac{U_{c}}{U_{m}}(m+\varepsilon) \text {. }
$$

As mentioned so far, the value of $\varepsilon$ decreases with increasing velocity if the frequency changes occur under a constant stage number. At a velocity range of $U_{m} \leq 11.3 \mathrm{~m} / \mathrm{s}$ and a stage number $m$ of 1 in Fig. 12, the experimental data at the velocity just before the frequency transition are included. From these data the average of the minimum of $\varepsilon$ is 0.15 . In addition employing 0.90 as the average of $U_{c} / U_{m}$ in Eq. (9), we find the Strouhal number to be 1.04, 1.94, and 2.84 for $m=1,2$, and 3, respectively. The solid lines corresponding to the constant Strouhal number indicated in Figs. 6 to 8 were obtained in this way. However further investigation is needed of these values, since estimation was made assuming that the minimum value of $\varepsilon$ was independent of stage number $m$, orifice spacing $l$, and velocity $U_{m}$.

From the foregoing the transition process of discrete frequency with velocity can be explained as follows. In Fig. 6, as the velocity increases gradually beyond $3.0 \mathrm{~m} / \mathrm{s}$, distance $\lambda$ between vortices increases due to the increment of convection velocity proportional to the flow velocity. Therefore the frequency of vortex shedding is increased to maintain a constant of $(l+h) / \lambda$; however, the increment of frequency is actually suppressed even lower due to the lock into the acoustic natural frequency. Thus in this velocity region, stage number $m$ is maintained to be 3, decreasing $\varepsilon$. As the value of $\varepsilon$ approaches lower limit with further increasing velocity, the frequency increases rapidly. Hence the decrease in $\varepsilon$ is suppressed, while the frequency greatly shifts from the acoustic natural frequency $(n=1)$. Consequently this condition can't be maintained steadily, so the transition of $m$ from 3 to 2 occurs at a velocity of $5.1 \mathrm{~m} / \mathrm{s}$ and the number of vortices decreases by one. Therefore, the frequency also decreases and again approaches the acoustic natural frequency, resulting in increasing $\varepsilon$. After phenomena similar to the above one recur with increasing velocity, stage number $m$ changes to 1 at $8.3 \mathrm{~m} / \mathrm{s}$, and the number of vortices decreases further by one. However, since the number of vortices can't become any smaller, the frequency jumps to $750 \mathrm{~Hz}$ near the third acoustic natural frequency at $11.3 \mathrm{~m} / \mathrm{s}$ with the consequence that $m$ returns to 3 and $\varepsilon$ recovers its previous value. Which value is taken after the frequency transition as a new stage number $m$ and an order of acoustic resonance mode $n$ is considered to be decided by the value of $\varepsilon$ for which the vortices can exist in a more stable state between two orifice plates. Furthermore it seems to be associated with the location of the second orifice plate as a sound source: whether the plate is located near a pressure node (in this experiment, $n=1,3$ and 4, as shown in Fig. 4) or a pressure loop ( $n=2)$. However this aspect remains future work.

Similar phenomena were also observed for orifice spacing $l=32$ and $17 \mathrm{~mm}$. Considering these results, $\varepsilon$ was found to vary over a range of $0<\varepsilon<0.5$. 

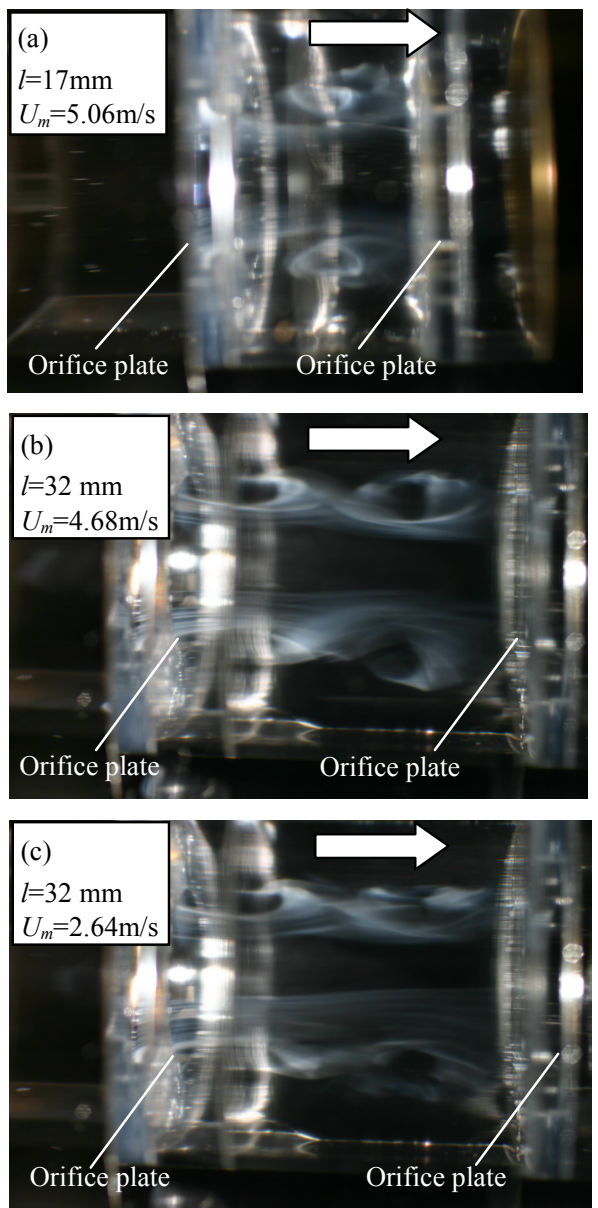

Fig. 15 Photographs using smoke visualization for the flows between two orifice plates

\subsection{Flow visualization}

Figure 15 shows photographs using smoke visualization for the typical flows between two orifice plates. Figure 15 (a) is taken under the condition of $l=17 \mathrm{~mm}$ and $U_{m}=5.06 \mathrm{~m} / \mathrm{s}$, and one distinct vortex ring is observed between two orifice plates. On the other hand stage number $m$ at this velocity is found to be 1 from the above Fig. 8. Figures 15 (b) and (c) show the photographs at $U_{m}=4.68$ and $2.64 \mathrm{~m} / \mathrm{s}$, respectively, for $l=32 \mathrm{~mm}$. In each figure, two [in Fig. (b)] or three [in Fig. (c)] vortex rings are generated, while the stage number corresponding to this figure is 2 or 3 as seen from Fig. 7. From these results the number of vortex rings was confirmed to correspond to the stage number.

\section{Conclusion}

The relationship between the velocity and the frequency of generated sound was scrutinized in a pipeline containing two closely spaced sharp-edged orifice plates. Moreover the convection velocity of the vortices was found by measuring the axial changes in the phase angle of the velocity fluctuation. The results are summarized as follows:

(1) The vortex shedding frequency is locked into the acoustic natural frequencies of the pipe mode and increases slightly with increasing velocity.

(2) The ratio of distance $l+h$ between two orifice plates to vortex spacing $\lambda$ can be expressed by Eq. (6). The value of $\varepsilon$ in Eq. (6) decreases with an increase in velocity over the range of $0<\varepsilon<0.5$, and as $\varepsilon$ reaches a critical value either a change in the number of vortices or a frequency jump occurs.

(3) The dimensionless convection velocity of vortices $U_{c} / U_{m}$ is 0.80 to 1.01 and $U_{c} / U_{\max }$ is 0.49 to 0.62 . 


\section{Acknowledgement}

The authors wish to express appreciation to Prof. K. Kuwabara of the Shizuoka Institute of Science and Technology for discussions and suggestions on flow visualization.

\section{References}

(1) v_BASE Forum, Generation of Self-Excited Vibration in LNG Pipelines (in Japanese), Proceedings of the D\&D '95 Conference of the Japan Society of Mechanical Engineers, No. 96-5 II (1996-8), pp. 82-83.

(2) Chanaud, R. C. and Powell, A., Srome Experiments concerning the Hole and Ring Tone, Journal of the Acoustical Society of America, Vol. 37, No. 5(1965), pp. 902-911.

(3) Nomoto, H. and Culick, F. E. C., An Experimental Investigation of Pure Tone Generated by Vortex Shedding in a Duct, Journal of Sound and Vibration, Vol. 84, No. 2(1982), pp. 247-252.

(4) Hourigan, K., Welsh, M. C., and Thompson, M. C., Aerodynamic Sources of Acoustic Resonance in a Duct with Baffles, Journal of Fluids and Structures, No. 4(1990), pp. 345-370.

(5) Harris, R. E., Weaver, D.S., and Dokainish, M. A., On the Generation of Acoustic Resonance in Pipelines, Proceedings of International Conference on Flow Induced Vibrations, (1987), pp. 543-550.

(6) Ramoureux, P. N. and Weaver, D. S., The Effects of Turbulence and Damping on Pipeline Acoustic Resonance, IMechE, C416/093, (1991), pp. 303-312.

(7) Huang, X. Y. and Weaver, D. S., On the Active Control of Shear Layer Oscillations across a Cavity in the Presence of Pipeline Acoustic Resonance, Journal of Fluids and Structures, No. 5(1991), pp. 207-219.

(8) Taniguchi, O. ed., Handbook of Mechanical Vibration (in Japanese), (1976), p. 1063, Yokendo.

(9) Rossiter, J. E., Wind-Tunnel Experiments on the Flow over Rectangular Cavities at Subsonic and Transonic Speeds, RAE Technical Report No. 64037(1964) and Reports and Memoranda No. 3438, (1964), pp. 1-34.

(10) Kurasawa H. et al., Self-Sustained Oscillation in an Axisymmetric Impinging Shear Layer, Transactions of the Japan Society of Mechanical Engineers, Series B, Vol. 53, No. 488(1987), pp. 1254-1261.

(11) Hussain, A. K. M. F. and Zaman, K. B. M. Q., The Free Shear Layer Tone Phenomena and Probe Interference, Journal of Fluid Mechanics, Vol. 87-Part 2(1978), pp. 349-383.

(12) Sarohia, V., Experimental Investigation of Oscillations in Flows over Shallow Cavities, AIAA Journal, Vol. 15, No. 7(1977), pp. 984-991.

(13) Hussain, A. K. M. F. and Zaman, K. B. M. Q., The Preferred Mode Coherent Structure in the Near Field of an Axisymmetric Jet with and without Excitation, Proceedings of Symposium on Turbulent Shear Flows, Toulouse, Springer, Berlin, (1981), pp. 390-401.

(14) Hussain, A. K. M. F. and Clark, A. R., On the Coherent Structure of the Axisymmetric Mixing Layer: a Flow-Visualization Study, Journal of Fluid Mechanics, Vol. 104(1981), pp. 263-294.

(15) Laufer, J. and Yen, T., Noise Generation by a Low-Mach-Number Jet, Journal of Fluid Mechanics, Vol. 134(1983), pp. 1-31. 